\title{
Toy Model for Molecular Motors
}

\author{
Hailemariam Ambaye und Klaus W. Kehr \\ Institut für Festkörperforschung, Forschungszentrum Jülich GmbH, D-52425 Jülich, Germany
}

(September 12, 2018)

\begin{abstract}
A hopping model for molecular motors is presented consisting of a state with asymmetric hopping rates with period 2 and a state with uniform hopping rates. State changes lead to a stationary unidirectional current of a particle. The current is explicitly calculated as a function of the rate of state changes, including also an external bias field. The Einstein relation between the linear mobility of the particle and its diffusion coefficient is investigated. The power input into the system is derived, as well as the power output resulting from the work performed against the bias field. The efficiency of this model is found to be rather small.

$05.40+$
\end{abstract}




\section{INTRODUCTION}

The modelling of molecular motors found great interest recently [1] 9]. The aim is the understanding of the working of molecular motors, where motor proteins perform unidirectional motion on structures having potentials without inversion symmetry. Several processes were proposed that may lead to unidirectional motion of particles in such potentials. One process is a switching of the potential between different states, which may be caused by chemical reactions [4, 10]. To understand the working of molecular motors in principle, simplified models are desired which are amenable to explicit calculations. In this note we present a very simple hopping model for unidirectional motion, which includes switching of the hopping potential between two states. Due to its simplicity, all quantities of interest can be calculated explicitly.

Recently two other simplified hopping models for molecular motors were introduced. Schimansky-Geier et al. [11] considered chains of segments that can switch either individually or collectively between two states. The segments consist of three sites with a non-symmetric arrangement of hopping rates in one state and uniform hopping rates in the other state. Kolomeisky and Widom [12] studied a model with coherent switching between two periodic non-symmetric potentials. In each potential only downward transitions are allowed; consequently the motion of a particle comes to rest in each state in the absence of switching events. Our model is simpler than the model of Schimansky-Geier in that the segments have only length 2 . We restrict the derivations to coherent switching between two states. The model is also different from the one of Kolomeisky and Widom in that it includes diffusion of the particle in the two states; unidirectional motion is then brought about by the state changes.

We restrict in this note the derivations to the stationary state with a unidirectional particle current; also we do not intend to model real molecular motors with our simplified model. In the following section the model is formulated in terms of its master equations. In section III the stationary current is calculated. The case with an additional uniform bias is treated in Sec.IV. The question of the validity of the Einstein relation between mobility and diffusion coefficient is addressed in Sec. V. Section VI presents a derivation of the power input and the power output when the systems performs work against the uniform bias field. Section VII contains concluding remarks.

\section{DEFINITION OF THE MODEL}

The model consists of a linear chain with sites to which hopping rates to the neighbor sites are associated. The chain exists in two different states with different hopping rates. The model is pictorially represented in Fig. 1 where the hopping rates are visualized by potentials. In the state (1) with an asymmetric potential, the sites with rates $\gamma_{1}, \delta_{1}$ are designated by 1 , and the ones with $\gamma_{2}, \delta_{2}$ by 2 . The transition to the other state (2) occurs in form of a Poisson process with rate $\Gamma_{f}$, and a particle on a site of type 1 or 2 is transferred to a site designated by 3 or 4 , respectively. In the second state with uniform potential all sites have transition rates $\gamma$ to the neighbor sites. The reverse transition to the non-symmetric state occurs with the same rate $\Gamma_{f}$. Periodic boundary conditions will be imposed on the system. 
To derive the current, it is sufficient to take a period 2. Displacements of the particle, however, have to be counted in the extended potentials.

Let $P_{1}(t)$ be the probability of finding the particle in any of the sites of type 1 in the chain in state $(1)$. The probabilities $P_{2}(t), P_{3}(t)$ and $P_{4}(t)$ are defined analogously. The probabilities fulfill the master equations

$$
\begin{aligned}
& \frac{d P_{1}}{d t}=\left(\gamma_{2}+\delta_{2}\right) P_{2}-\left(\gamma_{1}+\delta_{1}\right) P_{1}+\Gamma_{f}\left(P_{3}-P_{1}\right) \\
& \frac{d P_{2}}{d t}=\left(\gamma_{1}+\delta_{1}\right) P_{1}-\left(\gamma_{2}+\delta_{2}\right) P_{2}+\Gamma_{f}\left(P_{4}-P_{2}\right) \\
& \frac{d P_{3}}{d t}=2 \gamma P_{4}-2 \gamma P_{3}+\Gamma_{f}\left(P_{1}-P_{3}\right) \\
& \frac{d P_{4}}{d t}=2 \gamma P_{3}-2 \gamma P_{4}+\Gamma_{f}\left(P_{2}-P_{4}\right)
\end{aligned}
$$

The initial conditions are of no interest for the stationary state.

The sum of the four equation shows that the time derivative of the sum of the four probabilities vanishes. Hence $P_{1}+P_{2}+P_{3}+P_{4}=$ const $=1$. The sum of the first and the second master equation, and the sum of the third and fourth equation lead to the system of equations

$$
\begin{aligned}
& \frac{d\left(P_{1}+P_{2}\right)}{d t}=\Gamma_{f}\left(P_{3}+P_{4}\right)-\Gamma_{f}\left(P_{1}+P_{2}\right) \\
& \frac{d\left(P_{3}+P_{4}\right)}{d t}=\Gamma_{f}\left(P_{1}+P_{2}\right)-\Gamma_{f}\left(P_{3}+P_{4}\right) .
\end{aligned}
$$

This set of equations shows that the state changes occur with rate $\Gamma_{f}$. The stationary

solution of these equations is $P_{1}+P_{2}=P_{3}+P_{4}=\frac{1}{2}$ where we have also used the normalization of the sum of all probabilities.

\section{STATIONARY CURRENT}

The current from sites of type 1 to 2 , or from 3 to 4 , respectively, in the positive direction is given by

$$
J_{21}=\gamma_{1} P_{1}-\delta_{2} P_{2}+\gamma\left(P_{3}-P_{4}\right)
$$

The current from 2 to 1 , or from 4 to 3, respectively, in the positive direction is given by

$$
J_{12}=\gamma_{2} P_{2}-\delta_{1} P_{1}+\gamma\left(P_{4}-P_{3}\right) .
$$

In the stationary situation, the two currents are equal, $J_{21}=J_{12}=J$. Equating Eqs.(3) and (四) and using the stationary values for the sums $P_{1}+P_{2}$ and $P_{3}+P_{4}$, we obtain an equation relating the differences $P_{2}-P_{1}$ and $P_{4}-P_{3}$,

$$
\left(\gamma_{1}+\gamma_{2}+\delta_{1}+\delta_{2}\right) \frac{1}{2}\left(P_{2}-P_{1}\right)+2 \gamma\left(P_{4}-P_{3}\right)=\frac{1}{4}\left(\gamma_{1}-\gamma_{2}+\delta_{1}-\delta_{2}\right) .
$$

We find a second equation by subtracting the fourth from the third equation of the set (1), 


$$
\Gamma_{f}\left(P_{2}-P_{1}\right)-\left(4 \gamma+\Gamma_{f}\right)\left(P_{4}-P_{3}\right)=0 .
$$

The set of the two equations (5) and (6) for the two differences is readily solved. We proceed directly to the stationary current, which follows from the sum of Eqs. (3) and (伍) by inserting the differences, and the stationary values for the sums. The final result for the current is

$$
J=\frac{1}{2} \frac{\gamma \Gamma_{f}\left(\gamma_{1}+\gamma_{2}-\delta_{1}-\delta_{2}\right)}{\left(\gamma_{1}+\gamma_{2}+\delta_{1}+\delta_{2}\right)\left(4 \gamma+\Gamma_{f}\right)+4 \gamma \Gamma_{f}} .
$$

In the derivation of the current, there appears a term in the numerator proportional to $\gamma_{1} \gamma_{2}-\delta_{1} \delta_{2}$, see also Eq.(9) below. This term disappears due to the condition of detailed balance, which is valid within each of the two states of the chain. Consequently, the current is proportional to $\Gamma_{f}$ for small $\Gamma_{f}$, as it should be. We point out that the condition of detailed balance is fulfilled within each of the two states. The introduction of the state change with the rate $\Gamma_{f}$ leads to a violation of the condition of detailed balance when the full system is considered. This violation is necessary for the working of the motor, cf. the discussion in Ref. [10].

The result shows that a unidirectional current exists for finite values of the flip rate $\Gamma_{f}$. Figure 2 presents the result for a particular choice of the hopping rates on the chain, as a function of the flip rate $\Gamma_{f}$. The figure also contains the results of numerical simulations of the model. The current saturates for $\Gamma_{f}>>\gamma$ to the value

$$
J_{\Gamma_{f} \gg \gamma} \longrightarrow \frac{1}{2} \gamma \frac{\gamma_{1}+\gamma_{2}-\delta_{1}-\delta_{2}}{\gamma_{1}+\gamma_{2}+\delta_{1}+\delta_{2}+4 \gamma}
$$

From the continuum models with asymmetric potentials one expects that the unidirectional current is maximal for an optimal flip rate and vanishes in the limit of infinite flip rate. The saturation of the current at infinite flip rate is an artifact of our hopping model with only two sites. The model would correspond, in a continuum version, to a model containing sections with infinite slope in the potential. Models with at least three sites are required to remove the artifact of saturation. For instance, the model of Schimansky-Geier et al. 11] exhibits a maximum of the current as a function of the flip rate. The vanishing of the current in the limit of infinite flip rate, however, requires the presence of infinitely many sites in a segment, i.e., the continuum limit.

\section{UNIFORM BIAS}

It is also instructive to consider the influence of a uniform bias on the unidirectional motion of the particle. Then the system has to work against the bias field and the question is which bias is sufficient to stop the motion completely. A bias is introduced by multiplying the transition rates in state (1) in positive direction by a factor $b>1, \gamma_{1}, \gamma_{2} \rightarrow b \gamma_{1}, b \gamma_{2}$, and the transition rates in negative direction by $b^{-1}, \delta_{1}, \delta_{2} \rightarrow b^{-1} \delta_{1}, b^{-1} \delta_{2}$. In the state (2), the transition rates $\gamma$ are multiplied by $b$ for the positive direction and $b^{-1}$ for the negative direction. The bias factor is related to an external force $F$ by $b=\exp \left(F a / 2 k_{B} T\right)$ where $a$ is the lattice constant which is usually set unity.

The calculation of the stationary current is easily extended to the case with bias, using the modified transition rates as given above. The result is 


$$
\begin{aligned}
J & =\frac{1}{4 \Delta(b)}\left([ 2 ( b + b ^ { - 1 } ) \gamma + \Gamma _ { f } ] \left\{2 b^{2} \gamma_{1} \gamma_{2}-2 b^{-2} \delta_{1} \delta_{2}\right.\right. \\
& \left.+\left(b-b^{-1}\right) \gamma\left[b\left(\gamma_{1}+\gamma_{2}\right)+b^{-1}\left(\delta_{1}+\delta_{2}\right)\right]\right\} \\
& \left.+\left(b+b^{-1}\right) \gamma \Gamma_{f}\left[b\left(\gamma_{1}+\gamma_{2}\right)-b^{-1}\left(\delta_{1}+\delta_{2}\right)+2\left(b-b^{-1}\right) \gamma\right]\right)
\end{aligned}
$$

with

$$
\Delta(b)=\left[b\left(\gamma_{1}+\gamma_{2}\right)+b^{-1}\left(\delta_{1}+\delta_{2}\right)\right]\left[2\left(b+b^{-1}\right) \gamma+\Gamma_{f}\right]+2\left(b+b^{-1}\right) \gamma \Gamma_{f} .
$$

The result (17) is readily recovered for $b=1$.

The result for the stationary current as a function of the bias $b$ is presented in Fig.3, for one particular value of the flip rate $\Gamma_{f}$. One notices that the current behaves almost linearly as a function of $b$, for our choice of the parameters. The bias value where the current vanishes can be read from the figure. We note that this value is rather small. No analytic expression can be given because the condition $J(b)=0$ corresponds to an equation of $6^{\text {th }}$ order in $b$. The slope of the curve is given by

$$
\begin{aligned}
\left.\frac{d J}{d b}\right|_{b=1} & =\frac{1}{\Delta(b=1)}\left[\left(\gamma_{1} \gamma_{2}+\delta_{1} \delta_{2}\right)\left(4 \gamma+\Gamma_{f}\right)+\gamma\left(2 \gamma+\Gamma_{f}\right)\left(\gamma_{1}+\gamma_{2}+\delta_{1}+\delta_{2}\right)+2 \gamma^{2} \Gamma_{f}\right] \\
& -\frac{1}{2 \Delta(b=1)^{2}}\left[\gamma \Gamma_{f}\left(4 \gamma+\Gamma_{f}\right)\left(\gamma_{1}+\gamma_{2}-\delta_{1}-\delta_{2}\right)^{2}\right]
\end{aligned}
$$

Using the slope and the value of the current at $b=1$, an approximate prediction for the value of $b$ where the current vanishes can be obtained.

\section{MOBILITY AND DIFFUSIVITY}

The slope of $J(b)$ at $b=1$ gives a prediction for the linear mobility of a particle in the stationary situation, i.e., the increase/decrease of its velocity due to the action of a driving force. The question arises whether the linear mobility $B$ can be related to a diffusion coefficient $D$ by the Einstein relation $D=k_{B} T B$.

To examine this question, we calculated the diffusion coefficient of a particle in the system from its asymptotic mean-square displacement where the squared displacement is subtracted,

$$
<x^{2}(t)>-<x(t)>^{2} \longrightarrow 2 D t \quad \gamma t \rightarrow \infty
$$

To derive the mean-square displacement, site probabilities have to be considered that are

defined on a linear chain with $2 N$ sites, in the limit $N \rightarrow \infty$. The master equations (11) are easily extended to include site indices. The derivation of the mean-square displacement is a standard procedure, see for instance [13] or [14]. Because it is somewhat lengthy, it will not be reproduced here.

The result for the asymptotic diffusion coefficient is

$$
D=\frac{1}{\Delta(b=1)}\left[\left(\gamma_{1} \gamma_{2}+\delta_{1} \delta_{2}\right)\left(4 \gamma+\Gamma_{f}\right)+\gamma\left(2 \gamma+\Gamma_{f}\right)\left(\gamma_{1}+\gamma_{2}+\delta_{1}+\delta_{2}\right)+2 \gamma^{2} \Gamma_{f}\right]
$$


The diffusion coefficient is identical to the first term of the linear mobility as given in Eq.(11). Since the second term is missing, the Einstein relation between mobility and diffusion coefficient does not hold here (note that we are using units where $k_{B} T=1$ ). A violation of the Einstein relation is not surprising for the driven non-equilibrium, although stationary, system that is considered here. We point out that the numerical difference between the mobility and the diffusion coefficient is rather small for the parameters chosen here, it is barely visible on the scales of Figures 3 or 4.

Two limiting cases of the diffusion coefficient are of interest. First, for small flip rates $D$ can be brought into the form

$$
D\left(\Gamma_{f} \rightarrow 0\right)=\frac{\gamma}{2}+\frac{2}{\gamma_{1}^{-1}+\gamma_{2}^{-1}+\delta_{1}^{-1}+\delta_{2}^{-1}}
$$

This form of the diffusion coefficient follows from a two-state model where $D$ is deduced from a weighted superposition of the diffusion coefficients in state (2) (first term on the rhs of (14)) and of the coefficient in state (1) (second term). Since the flip rate is the same in both directions, the weights are $1 / 2$, and the result that is obtained agrees with (14) and is independent of the flip rate. Our derivations show that a two-state description is only applicable at small flip rates. For large flip rates, the diffusion coefficient saturates at

$$
D\left(\Gamma_{f} \rightarrow \infty\right)=\gamma-\frac{2 \gamma^{2}-\gamma_{1} \gamma_{2}-\delta_{1} \delta_{2}}{\gamma_{1}+\gamma_{2}+\delta_{1}+\delta_{2}+4 \gamma}
$$

We were not able to find a physical interpretation of this limiting behavior.

We also performed Monte Carlo simulations of the mean-square displacement of particles about the stationary state. The number of particles and system size used in the simulation are respectively $10^{4}$ and 4 with periodic boundary condition. Figure 4 demonstrates that $<x^{2}(t)>-<x(t)>^{2}$ is indeed proportional to $t$, and the diffusion coefficient $D$ can be deduced from the simulation results. The numerical results agree with the theoretical ones.

\section{POWER INPUT AND OUTPUT}

In this section we calculate the power input when the system is switched between two states, with an additional bias acting on the particle, and the power output, i.e., the work performed by the particle per unit time when it moves against the uniform bias.

Power is required to switch the system from the state with a uniform potential to the state with the non-symmetric potential. In our model, if a particle is on a site of type 4 in the uniform potential at the time where the switching occurs, its energy will be raised when it finds itself on site type 2 after the switching event. The energy follows from detailed balance in state (1),

$$
\beta \epsilon=\ln \frac{\delta_{2}}{\gamma_{1}}=\ln \frac{\gamma_{2}}{\delta_{1}}
$$

To obtain the power input, the average occupation of sites type 4 at the switching event is required. To derive this quantity, we consider the dynamics of the site occupation under the condition that the particle is remaining in state (1), or is remaining in state 
(2). The respective conditional probabilities, which will be called $Q_{1}(t), Q_{2}(t)$ for state $(1)$ and $Q_{3}(t), Q_{4}(t)$ for state $(2)$, obey analogous master equations as $P_{1}(t), \ldots, P_{4}(t)$, cf. (1) except that the terms proportional to $\Gamma_{f}$ are absent. The two uncoupled systems of master equations are readily solved and the solutions are

$$
\begin{aligned}
& Q_{1}(t)=A_{1}+B_{1} \exp \left[-\left(\gamma_{1}+\gamma_{2}+\delta_{1}+\delta_{2}\right) t\right] \\
& Q_{2}(t)=A_{2}+B_{2} \exp \left[-\left(\gamma_{1}+\gamma_{2}+\delta_{1}+\delta_{2}\right) t\right] \\
& Q_{3}(t)=A_{3}+B_{3} \exp (-4 \gamma t) \\
& Q_{4}(t)=A_{4}+B_{4} \exp (-4 \gamma t) .
\end{aligned}
$$

The constants are given as

$$
\begin{aligned}
& A_{1}=\frac{\gamma_{2}+\delta_{2}}{\gamma_{1}+\gamma_{2}+\delta_{1}+\delta_{2}} \\
& B_{1}=\frac{\left(\gamma_{1}+\delta_{1}\right) Q_{1}(0)-\left(\gamma_{2}+\delta_{2}\right) Q_{2}(0)}{\gamma_{1}+\gamma_{2}+\delta_{1}+\delta_{2}} \\
& A_{2}=\frac{\gamma_{1}+\delta_{1}}{\gamma_{1}+\gamma_{2}+\delta_{1}+\delta_{2}} \\
& B_{2}=-B_{1} \\
& A_{3}=A_{4}=\frac{1}{2} \\
& B_{3}=-B_{4}=\frac{1}{2}\left[Q_{3}(0)-Q_{4}(0)\right] .
\end{aligned}
$$

The strategy to obtain the average occupation probabilities of the four types of sites at the average switching times is as follows: We use as initial conditions for $Q_{i}(0)$ in the solutions of the uncoupled systems of master equations the complementary occupation probabilities $Q_{j}\left(t_{f}\right)$ in the other states at the mean switching time $t_{f}$, e.g., $Q_{1}(0)=Q_{3}\left(t_{f}\right)$. The mean switching time is given by $t_{f}=1 / \Gamma_{f}$. The coefficients for the solutions of the uncoupled master equations are then

$$
\begin{aligned}
& A_{1}=\frac{\gamma_{2}+\delta_{2}}{\gamma_{1}+\gamma_{2}+\delta_{1}+\delta_{2}} \\
& B_{1}=\frac{\left(\gamma_{1}+\delta_{1}\right) Q_{3}\left(t_{f}\right)-\left(\gamma_{2}+\delta_{2}\right) Q_{4}\left(t_{f}\right)}{\gamma_{1}+\gamma_{2}+\delta_{1}+\delta_{2}} \\
& A_{2}=\frac{\gamma_{1}+\delta_{1}}{\gamma_{1}+\gamma_{2}+\delta_{1}+\delta_{2}} \\
& B_{2}=-B_{1} \\
& A_{3}=A_{4}=\frac{1}{2} \\
& B_{3}=-B_{4}=\frac{1}{2}\left[Q_{1}\left(t_{f}\right)-Q_{2}\left(t_{f}\right)\right] .
\end{aligned}
$$

We now consider the solutions Eq.(17) at the time $t_{f}$ using the coefficients given in Eq.(19). They constitute sets of linear equations for the required coefficients $Q_{j}\left(t_{f}\right)$. The solutions of these equations are 


$$
\begin{aligned}
& Q_{1}\left(t_{f}\right)=\left[A_{1}-\frac{1}{2} \exp \left(-\Sigma t_{f}\right)+\frac{1}{2}\left(A_{2}-A_{1}\right) \exp \left[-\left(\gamma_{1}+\gamma_{2}+\delta_{1}+\delta_{2}\right) t_{f}\right]\right] / D N \\
& Q_{2}\left(t_{f}\right)=\left[A_{2}-\frac{1}{2} \exp \left(-\Sigma t_{f}\right)-\frac{1}{2}\left(A_{2}-A_{1}\right) \exp \left[-\left(\gamma_{1}+\gamma_{2}+\delta_{1}+\delta_{2}\right) t_{f}\right]\right] / D N \\
& Q_{3}\left(t_{f}\right)=\left[\frac{1}{2}-A_{1} \exp \left(-\Sigma t_{f}\right)-\frac{1}{2}\left(A_{2}-A_{1}\right) \exp \left(-4 \gamma t_{f}\right)\right] / D N \\
& Q_{4}\left(t_{f}\right)=\left[\frac{1}{2}-A_{2} \exp \left(-\Sigma t_{f}\right)+\frac{1}{2}\left(A_{2}-A_{1}\right) \exp \left(-4 \gamma t_{f}\right)\right] / D N
\end{aligned}
$$

where

$$
\begin{aligned}
\Sigma & =\gamma_{1}+\gamma_{2}+\delta_{1}+\delta_{2}+4 \gamma, \\
D N & =1-\exp \left(-\Sigma t_{f}\right) .
\end{aligned}
$$

We have verified by numerical simulations, where the system was switched at random times according to a Poisson process with mean flip time $t_{f}$, that the expressions given above describe the average occupations at the switching times. The above derivations were formally made for the case of no bias. A uniform bias is immediately included in the derivations if the transition rates $\gamma_{1}, \gamma_{2}$ are replaced by $b \gamma_{1}, b \gamma_{2}$, and the transition rates $\delta_{1}, \delta_{2}$ by $b^{-1} \delta_{1}, b^{-1} \delta_{2}$, respectively. The final formulae will be considered for the case including bias.

The power input is now obtained by multiplying the occupation of type 4 sites at the flipping time with the flipping rate and the energy supplied in one flip. This energy has been given in Eq.(16) in units of $k_{B} T$. Hence the power input is, in units of inverse time

$$
P_{\text {in }}=Q_{4}\left(t_{f}\right) \Gamma_{f} \epsilon / 2 k_{B} T .
$$

The power output is obtained from the velocity induced by the switching process by multiplying it with the force. The force is obtained from the bias parameter as

$$
F / k_{B} T=2 \ln (b)
$$

where the expression is dimensionless due to the division with $k_{B} T$ and the use of lattice constant $a=1$. Since the velocity is related to the current by a factor 2 in view of the normalization of 1 particle in the reduced system with periodic boundary conditions, we have

$$
P_{\text {out }}= \pm 2 J(F) F / k_{B} T .
$$

If the current for $F \rightarrow 0$ is negative for the choice of parameters used (as it is the case here) we compensate the sign by multiplying with -1 . The current is obtained as a function of $\mathrm{F}$ from Eq.(9) by using Eq.(23).

The power input, the power output and the efficiency of the motor, which is usually defined as the ratio of the power output to the input, are plotted in Fig.5, 6 and 7 respectively for three chosen flip rates as a function of the force acting on the system. The increment of the power input is so small that it cannot be seen from the figure. For example, for $\Gamma_{f}=0.2$ the power input increases from 0.19999336 to 0.19999401 in the range of the force considered, from 0 to 0.1 . The efficiency of the model that is studied here is rather small. This is related to the fact that the unidirectional current vanishes already for small bias fields opposing the motion of the particles, cf. Figs.3 and 6. In order to devise models that have a higher efficiency, more sites within a repeat unit are required, as well as proper adjustment of the parameters. 


\section{CONCLUDING REMARKS}

In this paper we have presented a very simple model for a molecular motor where a particle moves unidirectionally in a hopping potential which is flipping between a state having a potential without inversion symmetry and a state with uniform potential. Such models are intended to mimick motion of proteins in polar filaments [10]. The appeal of the model, which comprises only two different types of sites in the non symmetric state, is that all quantities of interest can be calculated explicitly. We demonstrated this by calculating the stationary current with and without bias which results from the switching of the system between the two states, and by deriving the power input and output of the system. The efficiency of the system as a motor is rather small for the parameters that were used. It was not the intention in any case to model real molecular motors. We also compared the linear mobility under the influence of a small bias field with the diffusion coefficient that follows from the mean-square displacement. We observed a violation of the Einstein relation between mobility and diffusion coefficient. We point out that we have a stationary nonequilibrium system where the question of the validity of the Einstein relation is nontrivial. The investigation of the fluctuations in these non-equilibrium systems appears to be of further interest.

\section{Acknowledgement}

We thank R. Sambeth for pointing out an error of a factor 0.5 in Eq.(22) to the paper. 


\section{REFERENCES}

[1] M. O. Magnasco, Phys. Rev. Lett. 71, 1477 (1993).

[2] R. D. Astumian and M. Bier, Phys. Rev. Lett. 72, 1766 (1994).

[3] C. R. Doering, W. Horsthemke, and J. Riordan, Phys. Rev. Lett. 72, 2984 (1994).

[4] J. Prost, J. F. Chauwin, L. Peliti, and A. Ajdari, Phys. Rev. Lett. 72, 2652 (1994).

[5] M. O. Magnasco, Phys. Rev. Lett. 72, 2656 (1994).

[6] J.F. Chauwin, A. Ajdari, and J. Prost, Europhys.Lett. 27, 421 (1994)

[7] H. X. Zhou and Y. D. Chen, Phys. Rev. Lett. 77, 194 (1996).

[8] P. Hänggi and R. Bartussek, in Nonlinear Physics of Complex Systems - Current Status and Future Trends, J. Parisi, S.C. Müller, and W. Zimmermann, Eds., Vol.476, 294 (Springer, Berlin, 1996)

[9] R.D. Astumian, Science 276, 917 (1997)

[10] F. Jülicher, A. Ajdari, and J. Prost, Rev.Mod.Phys. 69, 1269 (1997)

[11] L. Schimansky-Geier, M. Kschischo, and T. Fricke, Phys.Rev.Lett. 79, 3335 (1997)

[12] A.B. Kolomeisky and B. Widom, J.Stat.Phys., in press

[13] J.W. Haus and K.W. Kehr, Phys.Rep. 150, 263 (1987)

[14] K.W. Kehr, K. Mussawisade, and T. Wichmann, in Diffusion in Condensed Matter, J. Kaerger, P. Heitjans, and R. Haberlandt, Eds., Vieweg, Braunschweig, 1998, p. 265. 


\section{Figure Captions}

Fig. 1: Pictorial representation of the model for a molecular motor, with the transition rates indicated.

Fig. 2: Particle current without bias as a function of the flip rate $\Gamma_{f}$ between the two states. Line: analytical result, symbols: simulation results. The value of the transition rates used in the simulations are: $\gamma_{1}=\gamma_{2}=\gamma \exp (-2), \delta_{1}=\gamma \exp (-4), \gamma=\delta_{2}=0.5$. Transition from state (1) to state (2) and vice versa occurs with the rate $\Gamma_{f}$.

Fig. 3: Particle current as a function of the bias factor $b$ for a fixed flip rate, $\Gamma_{f}=0.5$. Line: analytical result, symbols: simulation results.

Fig. 4: Mean square displacement, where the squared mean displacement is subtracted, shown for different flip rates as a function of time. Symbols: $*$ simulation results for $\Gamma_{f}=5.0$; $\times$ simulation results for $\Gamma_{f}=0.5$; + simulation results for $\Gamma_{f}=0.1$; short dashes: theory for $\Gamma_{f}=5.0$; long dashes: theory for $\Gamma_{f}=0.5$; full line: theory for $\Gamma_{f}=0.1$.

Fig. 5: Results for the power input for three selected values of $\Gamma_{f}$. The corresponding values of the flip rates are indicated in the figure. Force is measured in units of $k_{B} T$

Fig. 6: Power output for three different values of $\Gamma_{f}$ in units of $k_{B} T$. The values of $\Gamma_{f}$ are indicated in the figure.

Fig. 7: Efficiency of the system vs force for three selected values of $\Gamma_{f}$, which are indicated on the curves. 


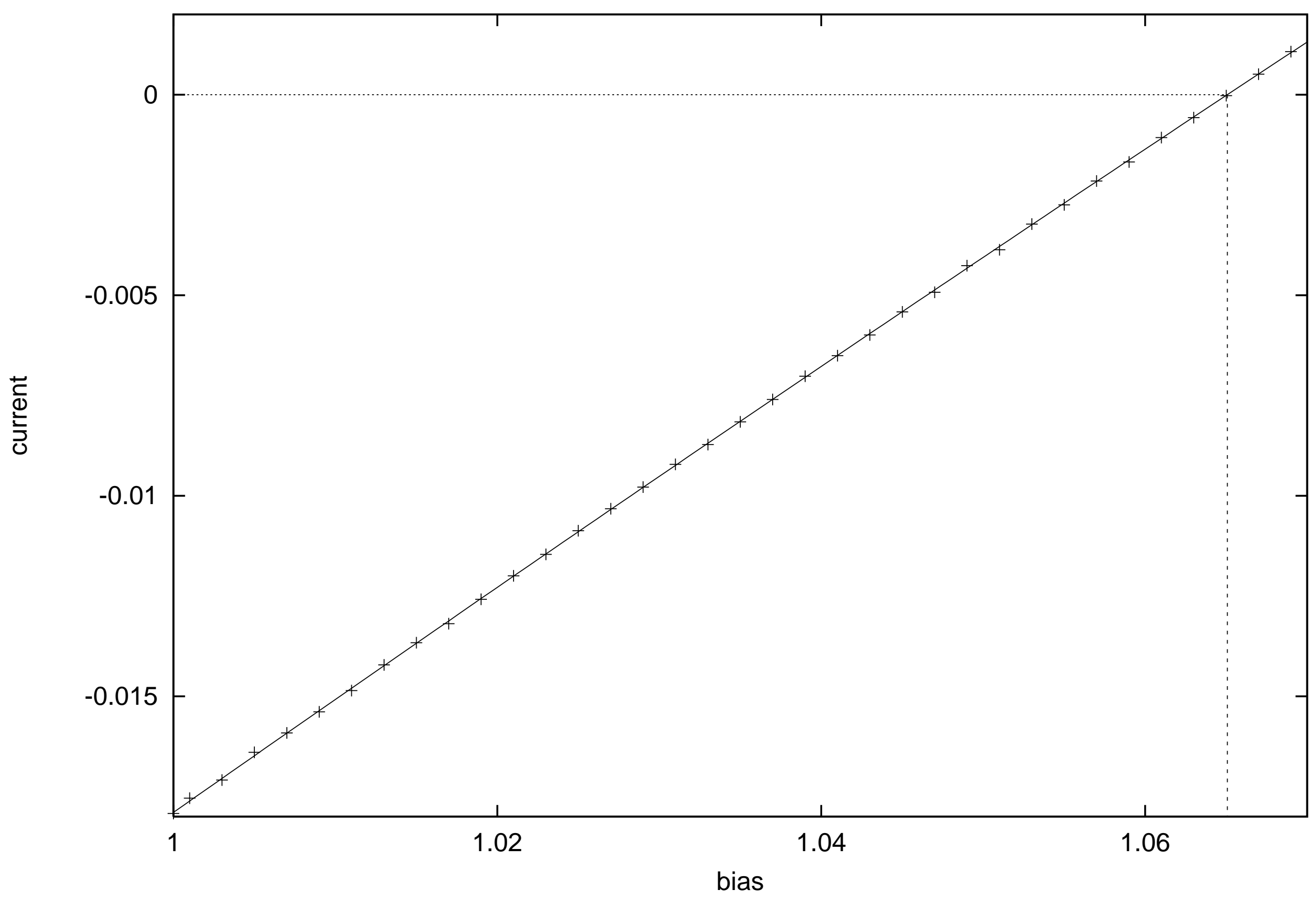




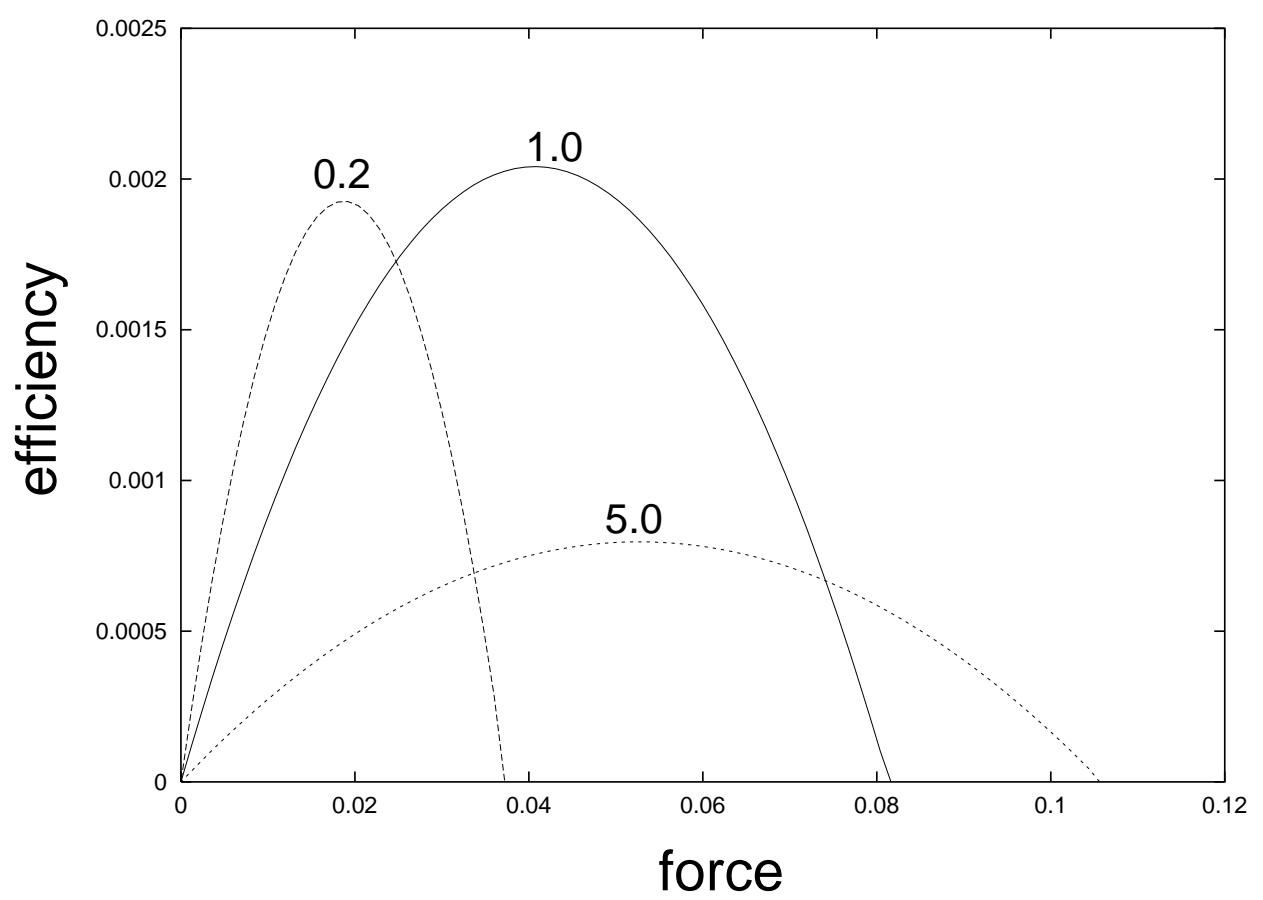




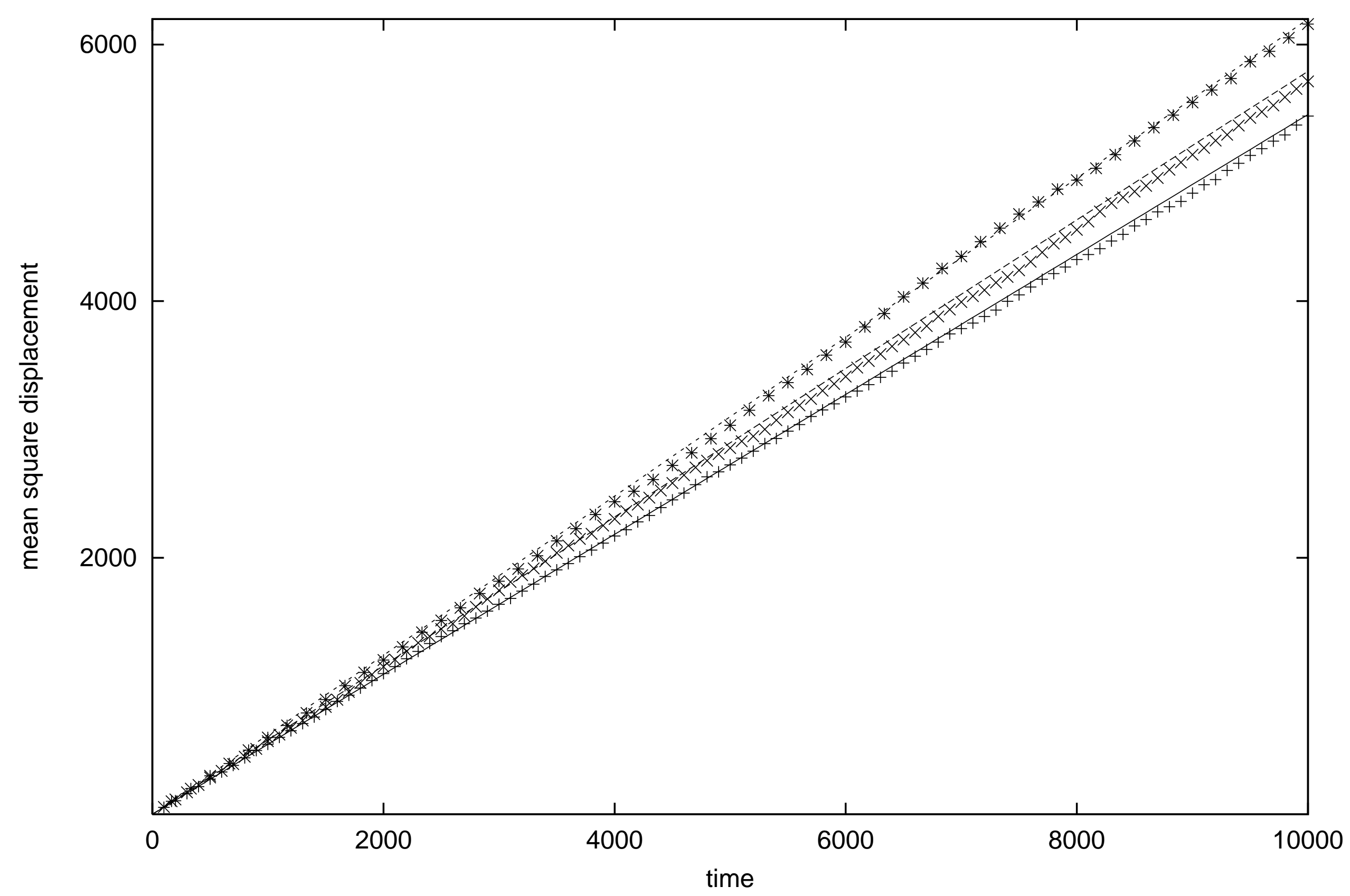




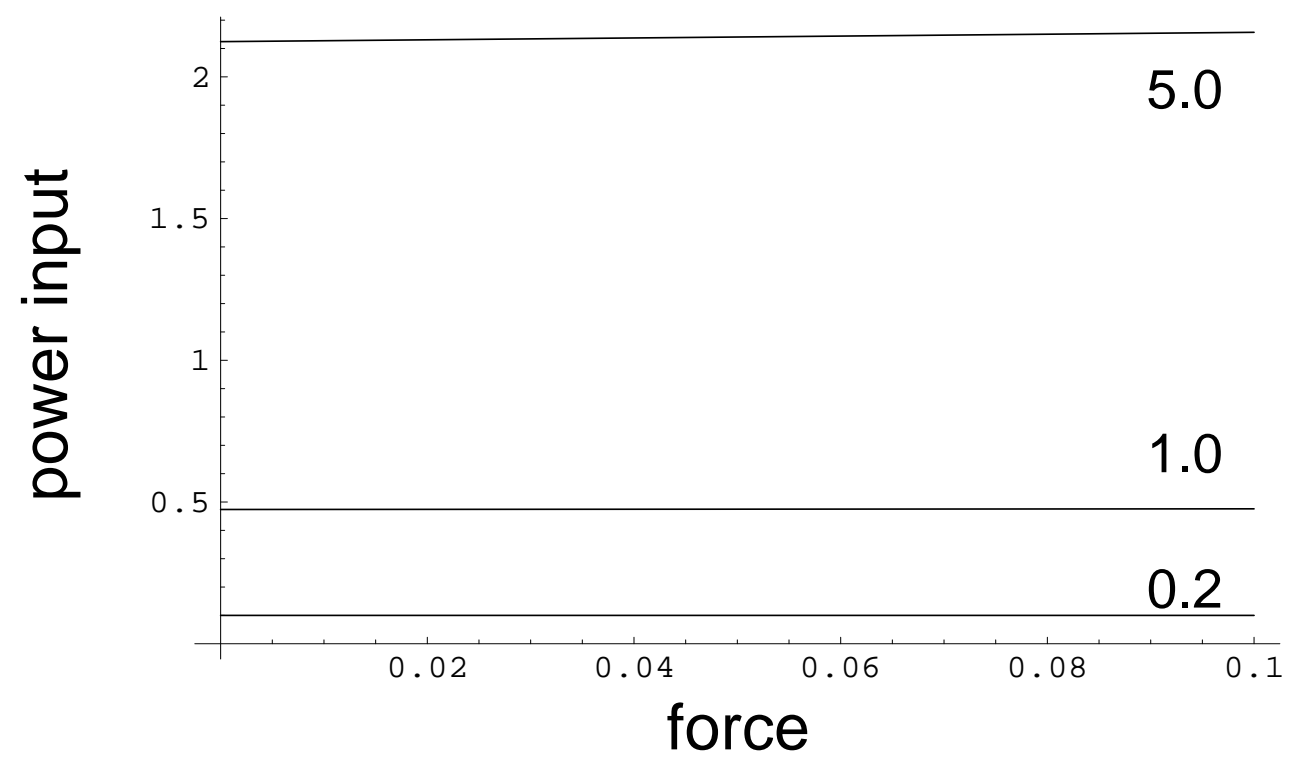




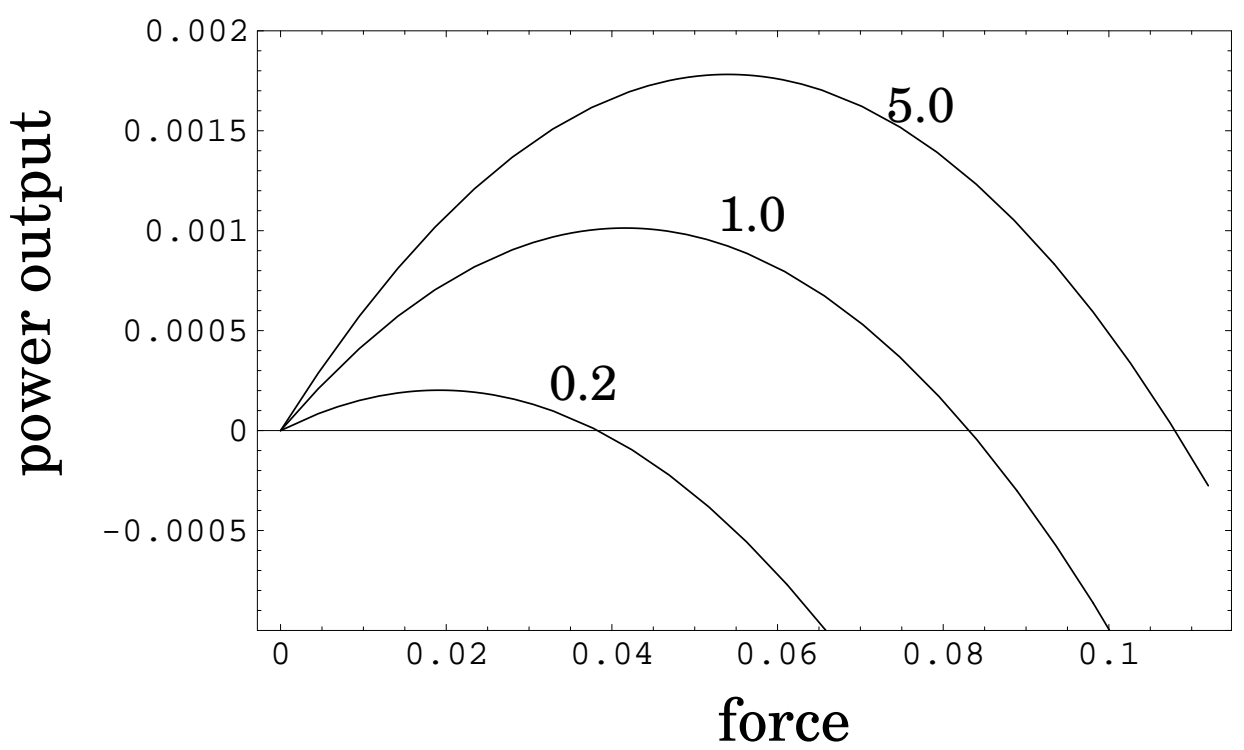




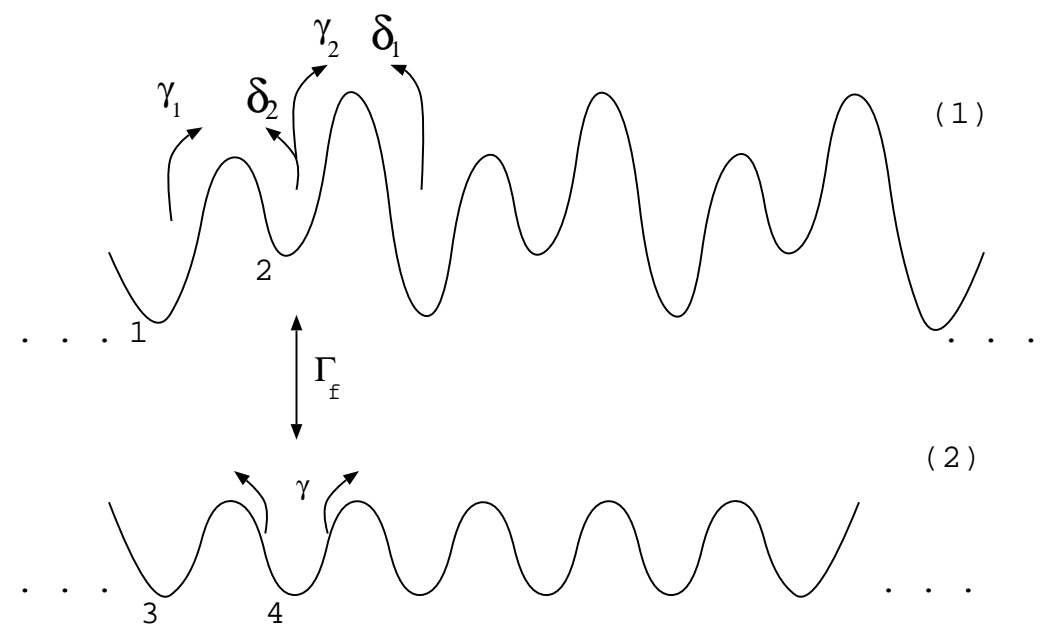




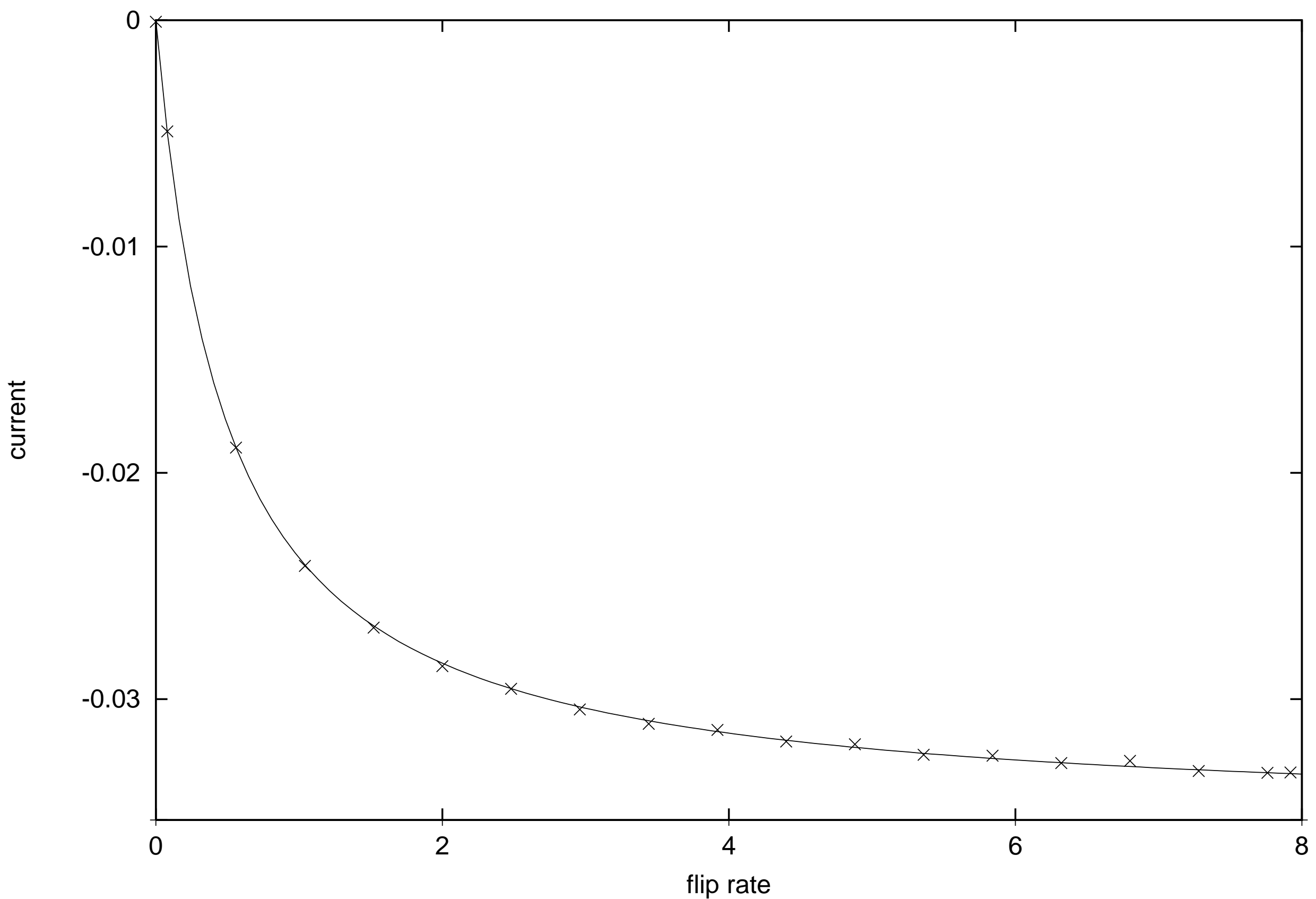

